\title{
Characterization of hollow fiber supported ionic liquid membranes using microfocus X-ray computed tomography
}

\author{
Sheng Dai ${ }^{\mathrm{a}}$, Yongkoo Seol ${ }^{\mathrm{a}}$, Shan Wickramanayake, ${ }^{\mathrm{a}, \mathrm{b}}$, and David Hopkinson ${ }^{\mathrm{a}^{*}}$ \\ ${ }^{a}$ National Energy Technology Laboratory, 3610 Collins Ferry Road, Morgantown, WV 26507, USA \\ ${ }^{\mathrm{b}}$ AECOM, 626 Cochrans Mill Road, Pittsburgh, PA 15236, USA \\ * Corresponding author. Tel.: +1 304285 4360; fax: +1 304285 4848. E-mail address: \\ david.hopkinson@netl.doe.gov (D. Hopkinson).
}

\begin{abstract}
This work demonstrates the use of X-ray computed tomography scanning to analyze the three dimensional structure of hollow fiber supported ionic liquid membranes (SILMs). In this type of gas separation membrane, the ionic liquid (IL) acts as the gas transport media, and therefore it is important to understand the distribution of ionic liquid within the hollow fiber support. Using the X-ray computed tomography technique, it was possible to obtain high quality three dimensional images in any plane or radial orientation of the membrane. Furthermore, and more importantly, quantitative data was determined for the density distribution and the pore distribution within the hollow fiber, as well as the distribution of pores filled with ionic liquid 1-hexyl-3-methylimidalzolium bis(trifluoromethylsulfonyl)imide $\left(\left[\mathrm{C}_{6} \mathrm{mim}\right]\left[\mathrm{Tf}_{2} \mathrm{~N}\right]\right)$. This data was used to characterize three types of hollow fibers: a) a dry fiber, b) a fiber that was fully saturated with ionic liquid, and c) a fiber that was saturated with IL and subsequently treated to achieve a selective layer with reduced thickness. Scans of the final type of fiber show that ionic liquid was isolated to the mesoporous outer wall region of the fiber cross section, which demonstrates the possibility of obtaining a thin, immobilized ionic liquid selective layer.
\end{abstract}

Keywords: hollow fiber; ionic liquid; pore distribution; X-ray; computed tomography 


\section{Introduction}

Supported ionic liquid membranes (SILMs) continue to be of interest for gas separations due to their high permeability and selectivity performance [1]. Because the gas transport medium of an SILM is a liquid layer suspended within a porous support, gas diffusivities tend to be relatively high compared with traditional dense polymer membranes. Further, the tunability and polar nature of ionic liquids can lead to high solubility for some gases, such as $\mathrm{CO}_{2}, \mathrm{SO}_{2}$, and water vapor. However, SILMs have yet to find their way into practical applications due to the inherent instability of a liquid membrane at high transmembrane pressures and because of the inability to control the membrane thickness. SILMs are commonly studied in laboratory settings in a flat sheet format for ease of preparation, handling and testing. In this case, the ionic liquid is allowed to fully saturate the support, and it is usually assumed that the selective layer thickness is equal to the thickness of the flat sheet support. These relatively thick supports result in a membrane with low permeance, despite the high permeability of the ionic liquid, and renders them impractical for applications.

Recently, some research groups have begun to develop techniques for implementing SILMs in hollow fiber format $[2,3]$. This opens the potential not only for building practical hollow fiber modules with large membrane surface area per unit volume, but also to take advantage of hollow fiber morphological features to reduce the selective layer thickness. In our previous work it was shown that a hollow fiber with an asymmetric distribution of pore sizes, ranging from relatively large macro-pores near the fiber bore to smaller meso-pores near the fiber shell, can be used to fabricate an SILM with a selective layer that does not span the entire thickness of the support [4]. This was achieved by first saturating the entire cross section of the hollow fiber support with ionic liquid, and subsequently flowing dry nitrogen gas through the fiber bore to remove liquid from the macro-pores. Meanwhile, liquid that is saturated into the meso-pores tends to remain in place because of the high capillary pressure that is associated with these small pore sizes [5].

In order to further optimize and reduce the thickness of these hollow fiber SILMs, it is important to understand the distribution of ionic liquid (IL) within the hollow fiber support. However, this remains a challenge due to the lack of characterization techniques which can be used to obtain high quality images or quantifiable data of the liquid location within the fiber cross section. Scanning electron microscopy (SEM) imaging tends to be ambiguous for several reasons: first due to the disturbance of the IL when a fiber is cut or fractured to prepare SEM samples; and second, the inability to locate and identify the IL. Relying on visual imaging alone is prone to interpretation, since an image may not clearly depict the difference between the solid and the liquid phases. By contrast, X-ray computed tomography (CT) scanning offers a non-destructive method for creating a three dimensional model of the fiber by detecting changes in density of the different phases of the sample. Whereas other microscopy techniques may be limited to flat, planar imaging, by using X-ray CT scanning different radial sections of a hollow fiber can be 'unrolled' and then used to calculate distributions of empty and liquid filled pores. This allows for a very versatile and comprehensive method to determine the location and distribution of IL within a fiber cross section.

X-ray CT scanning has been used in other studies of membrane morphology. Viguie and coauthors used X-ray CT scanning to analyze the distribution of macro-voids and macro-void initiation points in PVDF hollow fiber membranes [6]. The influence of air gap length and bore fluid solvent concentration on the macro-void shape and distribution was also analyzed. Manickam and McCutcheon compared mercury intrusion porosimetry, liquid extrusion porosimetry and X-ray CT imaging to obtain porosity and pore size data for polyester nonwoven membrane filters [7]. It was noted that porosimetry techniques can lead to a biased interpretation of porosity and pore size due to various effects including sample distortion, inability of the liquid to wet some pores, inability to detect blind pores, and an oversimplification of pore size data in the case of non-cylindrical pore geometries. Alternatively, X-ray CT 

low threshold contrast.

This paper presents a comprehensive morphological analysis of hollow fiber SILMs using X-ray CT scanning. Three fibers are compared, each consisting of the same materials and morphology but having different IL loadings and post-treatments. One fiber was untreated with IL and was used as the reference, a second fiber was fully saturated with IL, and a third fiber was saturated with IL and subsequently treated to achieve a selective layer with reduced thickness. The fibers were first imaged to show visual differences between them. The three dimensional CT images were then trimmed and unrolled at different radial locations to acquire density distribution profiles, pore size distributions, and distributions of liquid-filled pores. Finally, using the Young-Laplace model, an estimation of the residual ionic liquid profile was calculated based on the flow rate of gas through the fiber bore.

\section{X-ray CT Characterization}

\subsection{Materials}

Torlon ${ }^{\circledR}$ 4000T (Solvay Advanced Polymers), a polyamide-imide, was chosen as the polymeric material and N-methyl-2-pyrrolidone (NMP, from VWR) was used as the solvent to prepare the dope solution for fabrication of hollow fibers (neat Torlon density is $1.41 \mathrm{~g} / \mathrm{cm}^{3}$, structure is shown elsewhere [4]). An additive, $\mathrm{LiNO}_{3}$ (Sigma Aldrich), was also used to increase the fiber porosity. De-ionized water was selected as the non-solvent for the extraction of NMP. Methanol and hexane (VWR) were used during the solvent exchange to replace water from the hollow fibers. The hollow fiber pores were filled with the ionic liquid 1-hexyl-3-methylimidalzolium bis(trifluoromethylsulfonyl)imide $\left(\left[\mathrm{C}_{6} \mathrm{mim}\right]\left[\mathrm{Tf}{ }_{2} \mathrm{~N}\right]\right.$, from EMD Chemicals, density is $1.34 \mathrm{~g} / \mathrm{cm}^{3}$, structure is shown elsewhere [4]). The chemical structures of Torlon ${ }^{\circledR} 4000 \mathrm{~T}$, and $\left[\mathrm{C}_{6} \mathrm{mim}\right]\left[\mathrm{Tf}_{2} \mathrm{~N}\right]$ are shown elsewhere [2,4]. All of the membrane materials, solvents, non-solvents and additives were either in anhydrous form or of reagent grade.

\subsection{Hollow fiber fabrication}

Solid polymer materials were kept in vacuum for more than 24 hours at $120{ }^{\circ} \mathrm{C}$ to remove any absorbed moisture. The polymer dopes were prepared by dissolving polymer material and additives with NMP (14 wt $\%$ Torlon ${ }^{\circledR}, 4 \mathrm{wt} \% \mathrm{LiNO}_{3}, 82 \mathrm{wt} \% \mathrm{NMP}$ ) followed by mixing on a roller at room temperature. Once the mixing process was completed, the polymer dope was transferred into a vacuum oven at room temperature and left until all of the dissolved gas bubbles disappeared. Later, the polymer dope was transferred to a syringe pump (Teledyne ISCO) and kept undisturbed at room temperature and under vacuum for another 24 hours to de-gas the polymer solution further. A mixture of water and NMP (4:96 wt \%), which was used as the bore fluid, was loaded into another syringe pump and kept undisturbed at room temperature for 24 hours to de-gas. The composition of the bore fluid was determined using the cloud point technique [8].

Hollow fibers were fabricated as explained elsewhere using a single-layer spinneret at ambient conditions $[2,9,10]$. Syringe pumps were used to control the bore and sheath fluid flow rates at 60 and 90 $\mathrm{mL} /$ hour, respectively, and an air gap of $2 \mathrm{~cm}$ was used for all experiments. A water container with a 2.2 $\mathrm{m}$ long non-solvent quench path was used for the phase separation. Upon entering the quench bath, the nascent fibers were phase separated and collected on a winding drum at a speed of about $6.75 \mathrm{~m} / \mathrm{min}$. The residual NMP solvent in the fibers has the ability to plasticize the solidified polymer wall causing shape deformation. Therefore, collected fibers were rinsed in de-ionized water three times for 24 hours each time. The fibers were then rinsed in methanol three times for 30 minutes each time. Finally the fibers were rinsed with hexane three times for 30 minutes each time. After rinsing, fibers were transferred into a vacuum oven and dried at $30{ }^{\circ} \mathrm{C}$ for 24 hours. 
Three types of fibers were prepared for X-ray CT scanning. The first fiber was untreated with ionic liquid and used as the reference. The second fiber was immersed in $\left[\mathrm{C}_{6} \mathrm{mim}\right]\left[\mathrm{Tf}_{2} \mathrm{~N}\right]$ for 48 hours in vacuum to ensure that all pores were completely filled with ionic liquid [2]. The third fiber was soaked in ionic liquid for 48 hours in vacuum and subsequently dry nitrogen gas was flowed through the fiber bore at 1.5 standard liters per minute (SLPM) for 3 hours in order to remove excess ionic liquid. Although no gas permeance testing was conducted in this study, this procedure is normally necessary to use hollow fiber SILMs for gas separations. Otherwise, excess IL can clog the fiber bore [2].

\subsection{Methodology: X-ray CT}

The theoretical foundation of X-ray CT is to measure the X-ray attenuation by a sample situated in the beam pathway. For scanning, X-ray photons are generated from a source, attenuated when penetrating the sample, and the attenuated beams are then collected by a detector. The intensity of X-rays decays exponentially while passing through an object, as described by (or Beer-Lambert law):

$I=I_{0} e^{-\alpha L}$,

where $I$ is the X-ray intensity after passing through the scanned object, $I_{0}$ is the X-ray intensity emitted by the source, $L$ is the length of the object that X-rays passed through, and $\alpha$ is the linear attenuation coefficient. This linear attenuation coefficient is inherently determined by the X-ray energy $E$, the material density $\rho$, and the atomic number $N$ of the object:

$$
\alpha=\rho\left(a+b \frac{N^{3.8}}{E^{3.2}}\right)
$$

where $a$ and $b$ are parameters capturing the effects of Compton scattering and photoelectric absorption respectively $[11,12]$.

The attenuated X-ray photons captured by the detector for each scan form a radiograph or projection. A collection of projections at different angles in a full rotation are then processed for a three dimensional (3D) reconstruction, which is also known as a back projection [13]. The reconstructed 3D image is composed of linear attenuation coefficients (or voxel intensity) for the sample and this spatial distribution of voxel intensity captures the internal structure of the sample. Therefore, the voxel intensity is inherently the averaged linear attenuation coefficient of the smallest $3 \mathrm{D}$ volume that can be resolved, i.e., resolution. The micro-focus CT can visualize, characterize, and analyze multiphase samples at micron level of voxel resolution.

\subsection{CT scanning of the hollow fiber}

An Xradia Micro XCT-400 scanner (Zeiss) was used in this study. This scanner can achieve submicron resolution with $0.7 \mathrm{~mm}$ field of view in all three dimensions. Fiber samples were held vertically within a plastic tube with $1 \mathrm{~mm}$ inner diameter. Detailed scanning parameters are listed in Table 1.

Table 1. CT scanning parameters.

\begin{tabular}{lll}
\hline Scanning Parameters & Unit & Value \\
\hline Source-object distance & $\mathrm{mm}$ & 44.57 \\
Detector-object distance & $\mathrm{mm}$ & 9.55
\end{tabular}


No. of scans per degree of angle Pixel size

Three types of hollow fiber samples were scanned: dried, saturated by IL, and after being cleaned by nitrogen gas. We use 'dry', 'IL saturated', and 'cleaned' hereafter to describe these three fiber samples. Figure 1 shows the slice view of obtained CT images for these three types of samples. The resulting resolution of the scans was 1.11 microns. CT scans were reconstructed manually to minimize beam hardening and pixel shift artifacts using XMReconstructor (Xradia, Inc). It can be clearly seen that all pores are filled with IL in the saturated fiber (Figure 1b). In the cleaned fiber the macro-pores that are open to the bore become evacuated, but other macro-pores retain some residual IL (Figure 1c).

(a)

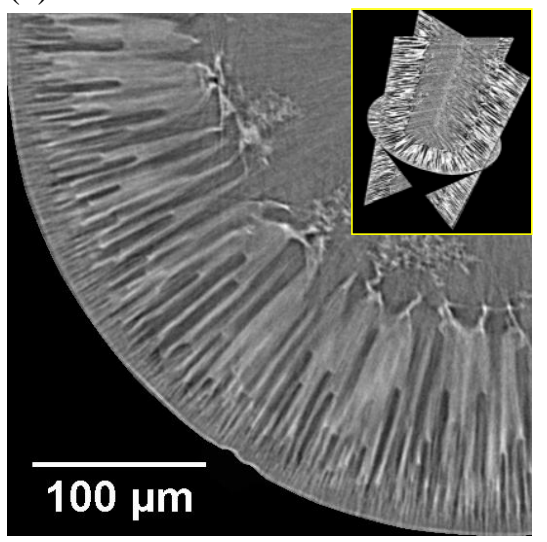

(b)

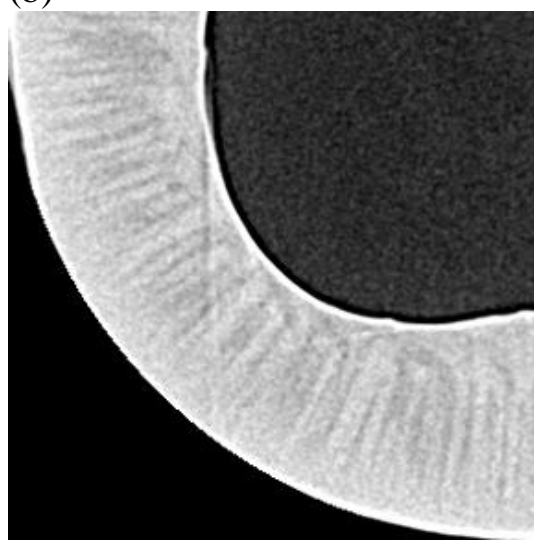

(c)

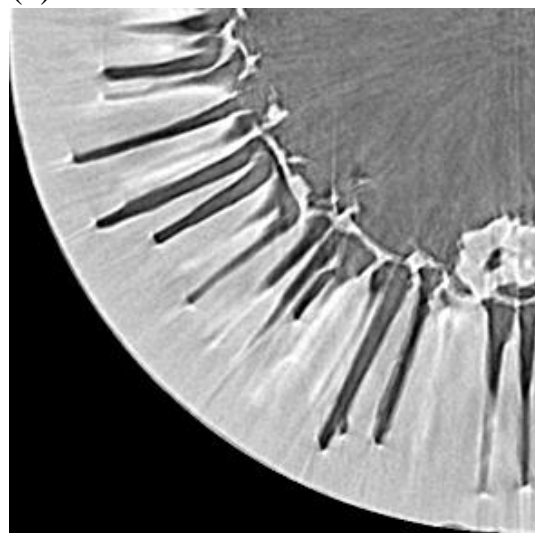

Fig. 1. Micro-CT scanning images: slice view of (a) dry, (b) IL saturated, and (c) cleaned Torlon ${ }^{\circledR}$ fiber. The inset in (a) shows a 3D orthoslice view of the dry fiber, showing sectional views of three perpendicular planes. The scale is the same for (a), (b), and (c).

\subsection{CT image processing}

Reconstructed 3D CT images were further processed using a public domain image processing and analysis software Image ${ }^{\circledR} 1.47$ [14]. The fibers of interest in this study are cylindrical and hollow; thus, all spatially varying properties are expressed in a polar coordinate. The morphological and physical properties, such as pore size distribution and liquid saturation, at any polar distance $x$ of the 3D fiber CT images are analyzed using an unrolling technique (Figure 2). In order to process the CT images, the stack of 2D slice images is first trimmed by a concentric circle with a radius $x$ and only the content within this circle remains. Then, the trimmed 2D slice images are reconstructed for a 3D fiber structure, the cylindrical surface of which shows the internal structure of the fiber at a radial distance of $x$ (Figure $2 b$ ). To obtain the surface information of this 'trimmed' fiber, we use the polar transformation plugin algorithm to unroll the surface [15], converting the polar space into Cartesian coordinates, as shown in Figure 2c. Therefore, the internal structure of the 3D fiber at any radial distance is captured and presented by a $2 \mathrm{D}$ unrolled image. More details on techniques of CT image processing are available in other literature [16-19].

\begin{tabular}{|l|l|l|}
\hline (a) & (b) & (c) \\
\hline
\end{tabular}



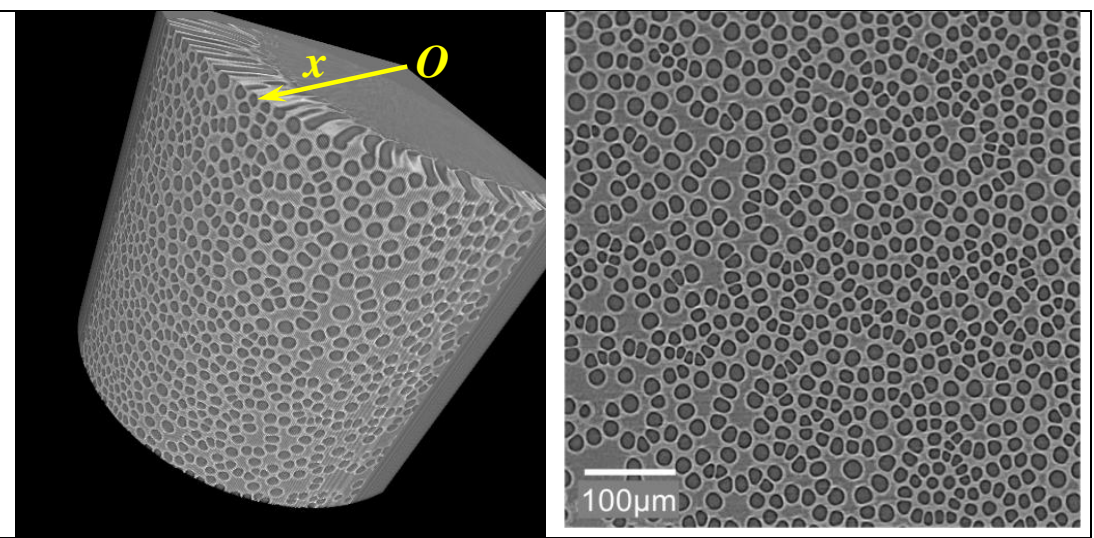

Fig. 2. Illustration of image processing methodology: unrolling the fiber at any position of interest. (a) Slice view illustrating a radial position $x$ from the center of the fiber, $O$. (b) 3D view of the fiber trimmed at the radial distance $x$. (c) Unrolled surface of 3D fiber showing pore characteristics.

\section{X-ray CT Results}

\subsection{Dry fiber pore distributions}

The hollow fiber morphology studied here consists of a macro-porous region at the inner cross section and a meso-porous region at the outer wall (refer to Figure 1a). This morphology is advantageous for a hollow fiber SILM application, since the high capillary pressure of the meso-porous region promotes trapping of the IL. Conversely, the larger macro-pores allow IL to be released during the cleaning procedure, which limits the thickness of the selective layer to the meso-pore region. The pore characteristics of the dry fiber are analyzed using the unrolling technique introduced earlier.

For the convenience of this description, we here introduce a dimensionless radial distance $\lambda=x / R$, where $x$ is the radial distance from the center and $R$ is the radius of fiber; so $\lambda=0$ means the center and $\lambda$ $=1$ represents the outside boundary of the fiber (note that the dry hollow fiber in this study has an outer radius $R=309 \mu \mathrm{m}$ ). Figure 3a illustrates the pore structures at various radial locations of the fiber, i.e., $\lambda$ $=0.7,0.8,0.9$, and 0.99 from inside out. The distributions of the pore area at different radial locations of the fiber are shown in Figure $3 \mathrm{~b}$. As a general trend, the pores become smaller and have narrower range of distribution closer to the outside surface of the fiber.

(a) 


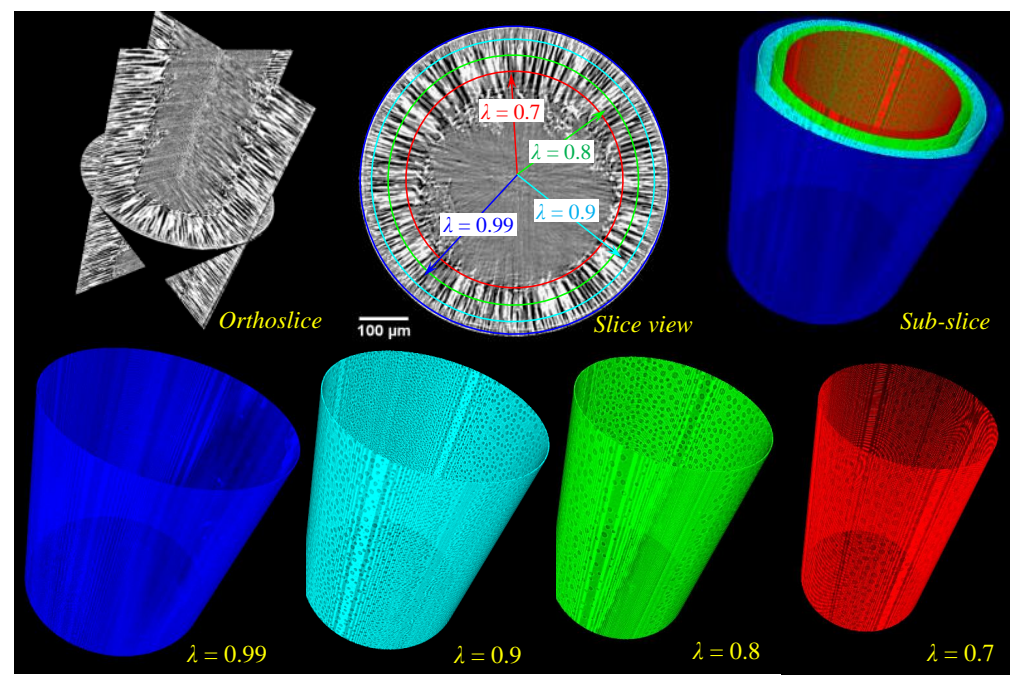

(b)

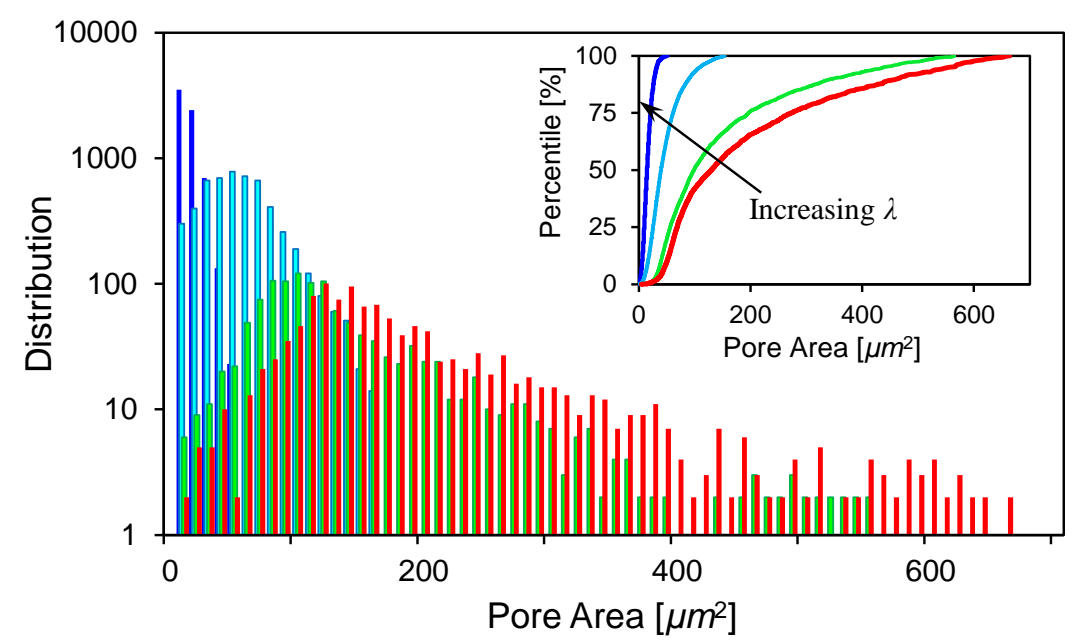

Fig. 3. Pore size distributions at different locations of interest in the fiber. (a) The fiber is trimmed at different radial distances $\lambda=x / R$ (refer also to Figure 2a). (b) Corresponding pore size distributions at $\lambda=$ $0.7,0.8,0.9$, and 0.99 . The inset shows cumulative pore area distributions.

\subsection{Density profile of fibers with IL}

Pure materials with known densities were scanned under identical scanning conditions of the fiber for CT density calibration (see details in [20]). Obtained density profiles of the three different fiber types are shown in Figure 4. Note that these density values are the averaged densities of different material components. For instance, the density profile of the cleaned fiber reflects the volume averaged densities of Torlon ${ }^{\circledR}$, IL, and air at different radial locations. Therefore, with known material densities of each component, we can inversely infer the volume fraction of each component at various locations. The density of the dry fiber increases sharply after $\lambda>\sim 0.9$, suggesting a rapid decrease in void fraction. The saturated fiber maintains a relatively high density throughout the solid portion of its cross section, indicating that the majority of pores are in fact saturated with ionic liquid. The cleaned fiber has roughly the same density profile as the dry fiber at $\lambda<\sim 0.75$, and a higher density at $\lambda>\sim 0.75$, indicating that IL is retained only in the outer meso-porous region of the fiber after the cleaning procedure. 


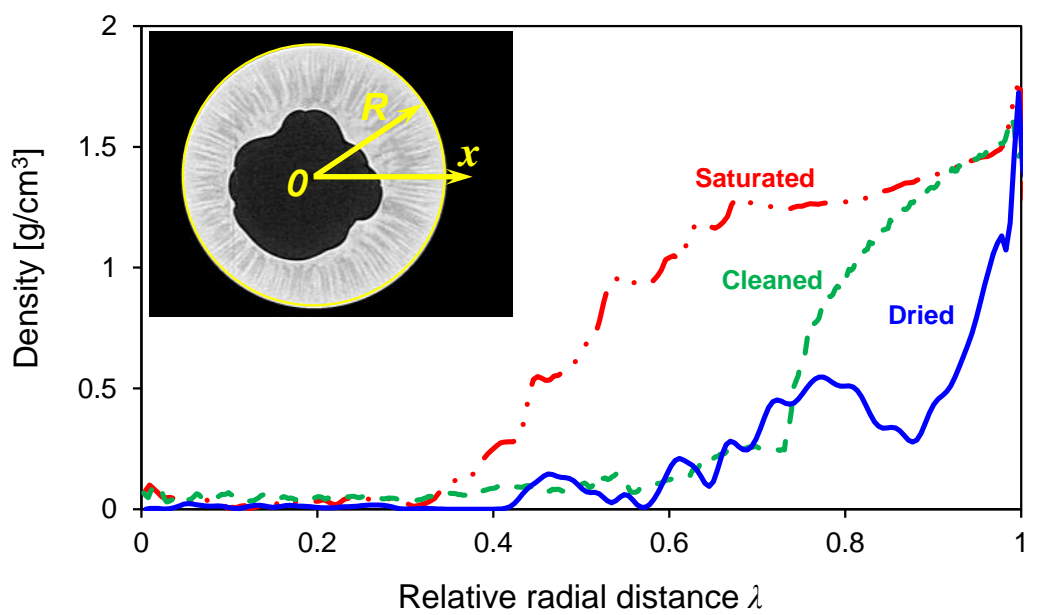

Fig. 4. Density profiles of dry, IL saturated, and cleaned fibers.

\subsection{Residual IL saturation}

The unrolled images of meso- and macro-pores (at $\lambda=0.99$ and 0.8 respectively) in fibers with dried, IL saturated, and gas cleaned conditions are shown in Figure 5a. Clearly, the pore sizes near the outside boundaries $(\lambda=0.99)$ are much smaller than the inner ones $(\lambda=0.8)$. Since air and IL have different densities and atomic numbers, the CT numbers (i.e., gray values in CT images) of these two phases are different. To obtain the IL distribution in the saturated and cleaned fibers, we use the Otsu method $[16,21,22]$ to threshold the unrolled CT images and obtain the proportion of gas and IL phases within the pores. As shown in Figure 5b, the distribution of IL-filled pores in the meso-pore region $(\lambda=$ $0.99)$ is almost identical for the saturated and cleaned fibers; while in the macro-pore region $(\lambda=0.8)$, the cleaned fiber only retains IL in relatively smaller pores. Smaller pores have higher capillary pressure which prevents the IL from being purged by the nitrogen gas. Therefore, the macro-pores become partially saturated after gas cleaning while the meso-pores stay almost saturated.

(a)

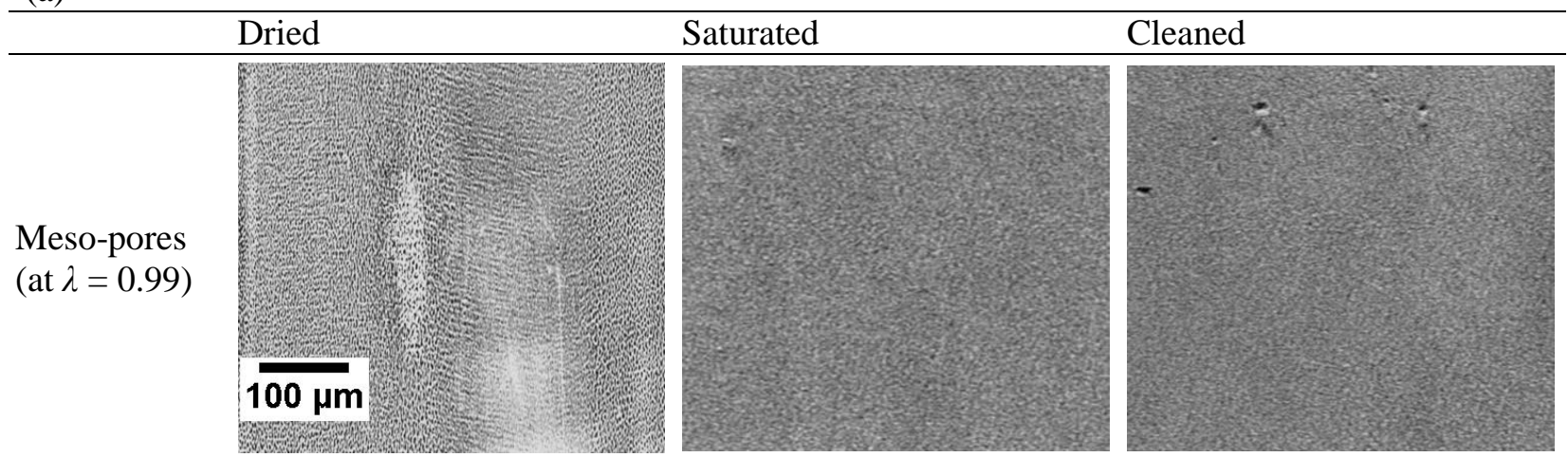


Meso-pores (at $\lambda=0.8$ )
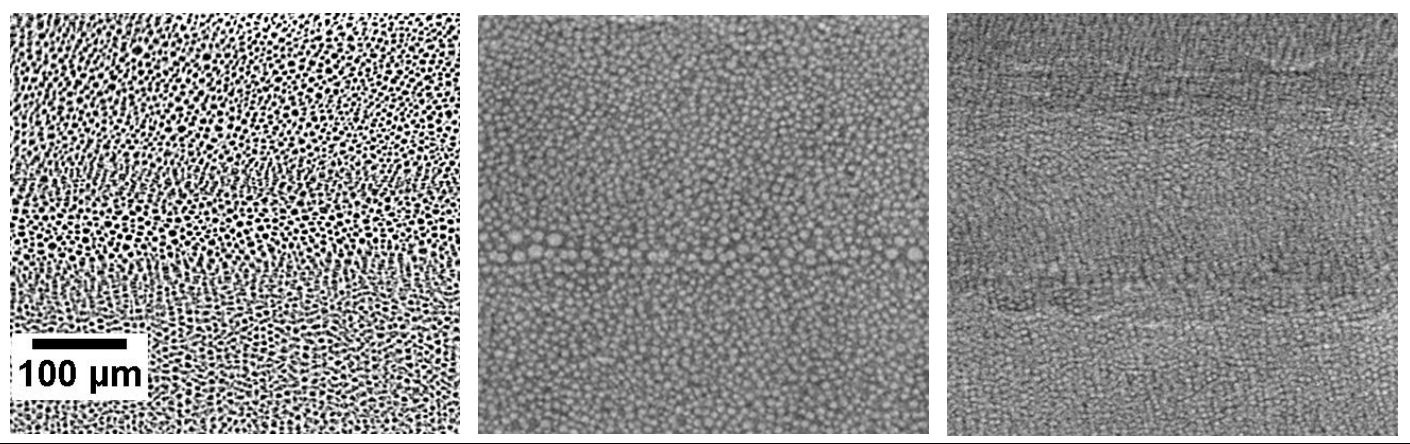

(b)
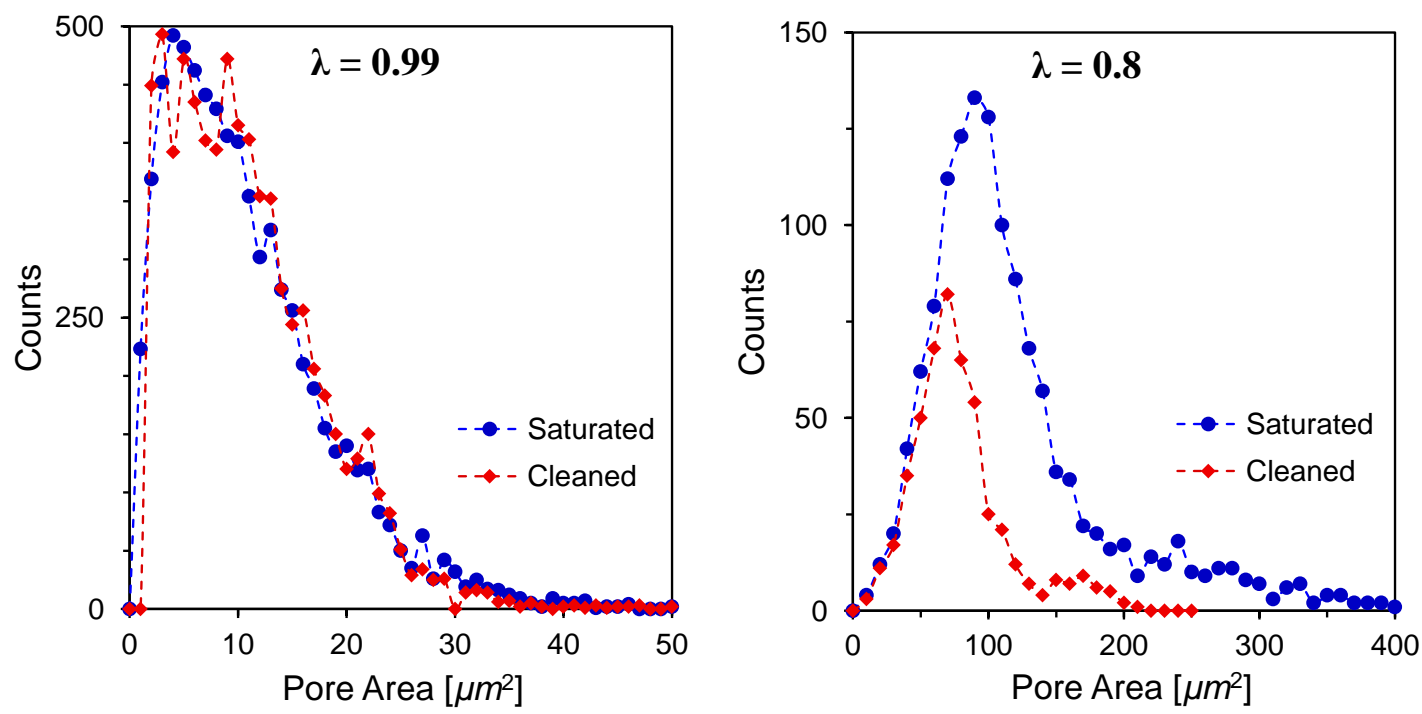

Fig. 5. Meso- and macro-pores of the fiber for dry, IL saturated, and cleaned conditions. (a) Unrolled surface view of the meso- and macro-pores. (b) Distributions of pore area of the IL-filled pores in the saturated and cleaned fibers.

The porosity and IL-saturation (i.e., IL volume divided by pore space) profiles are obtained by threshold analysis of the unrolled CT images throughout the entire range of radial positions (Figure 6). The porosity generally decreases towards the outside boundary of the fiber. Since the fiber is hollow, the porosity at the center $(\lambda=0)$ is virtually 1 . This value starts to decrease at $\lambda \approx 0.55$, reaches a stable value of $v \approx 0.45$ when $\lambda \approx 0.65-0.9$, and then decreases again as moving toward the meso-pore region, the porosity of which is $v \approx 0.3$. These porosities are consistent with a previous study in which the porosities of the macro-pore region and of the meso-pore region were determined by threshold analysis of SEM images for the same type of fiber ( $v_{\text {macro }} \approx 0.52$ and $v_{\text {meso }} \approx 0.29$ ) [4]. Thus, at the outside boundary of the fiber, the pores decrease not only in size but also in volume fraction. Inversely, the residual IL saturation increases towards the fiber's outside boundary, primarily due to increased capillarity to retain the IL within the pores. 


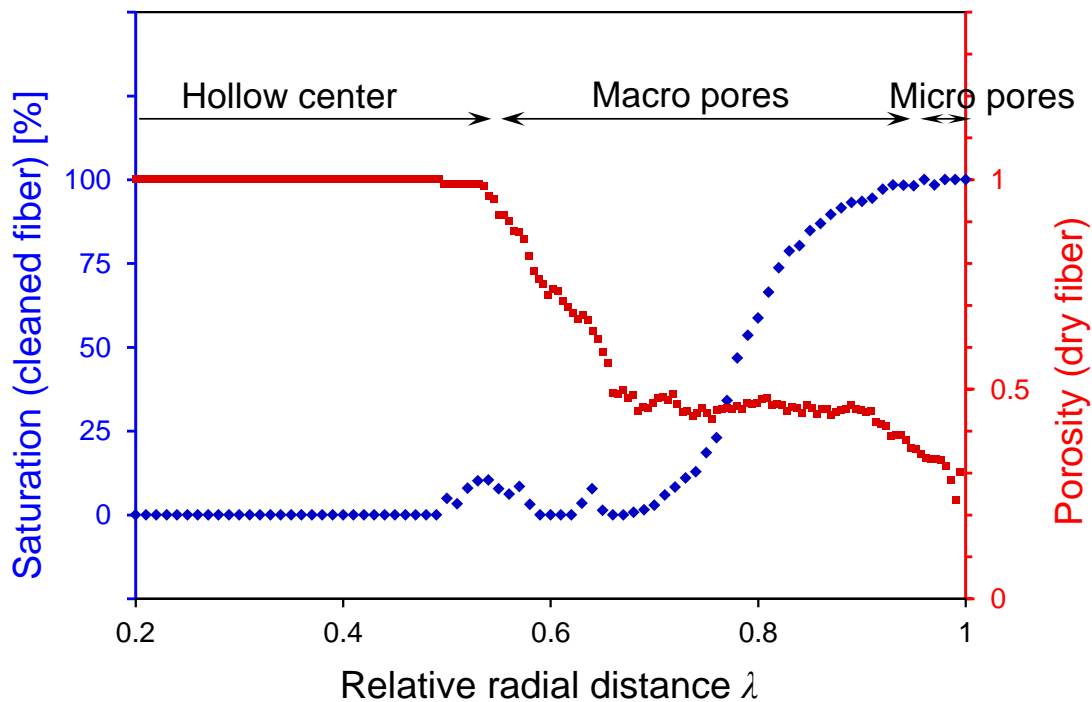

Fig. 6. Radial profiles of average porosity in the dry fiber (right axis) and residual IL saturation in the cleaned fiber (left axis). The average porosity gradually decreases from inside out. However, the residual IL saturation profile shows an inverse trend.

\section{Analyses and Discussion}

\subsection{Pore size characteristics}

Figure 7a shows the distribution histograms of the pore diameter $d_{p o r e}$ at several locations. The pore diameter $d_{\text {pore }}$ is computed directly from the measured pore areas $A_{\text {pore }}$ assuming a circular pore shape, i.e., $d_{\text {pore }}=\sqrt{4 A_{\text {pore }} / \pi}$. The macro-pore diameters (at $\lambda=0.7,0.8$, and 0.9 ) follow a normal distribution $N\left(\mu, \sigma^{2}\right)$, where $\mu$ is the mean and $\sigma^{2}$ is the variance. The macro-pore distributions have an identical standard deviation of $\sigma=2 \mu \mathrm{m}$ (or variance $\sigma^{2}=4 \mu \mathrm{m}^{2}$ ), while the meso-pore (at $\lambda=0.99$ ) distribution has a smaller standard deviation of $\sigma=1.2 \mu \mathrm{m}$, indicating less scatter in pore size. In fact, the pore size distribution of the meso-porous region can be better characterized by a log-normal distribution $\ln N(\mu, \sigma)=\ln N(0.36,0.2)$, with mean $e^{\mu+\sigma^{2} / 2}=1.46 \mu \mathrm{m}$ and variance $\left(e^{\sigma^{2}}-1\right) e^{2 \mu+\sigma^{2}}=0.3 \mu \mathrm{m}^{2}$ (see the insert). Note that the pixel size of the CT images is $1.11 \mu \mathrm{m}$ so that any pores smaller than this scale cannot be resolved. In general, the mean pore diameter $\mu_{\text {dpore }}$ decreases with increasing normalized radial distance $\lambda$. This relation can be characterized as: $\mu_{d_{\text {pore }}}[\mu m]=31.5-30 \lambda$ (see Figure $7 \mathrm{~b}$ ).

(a) 

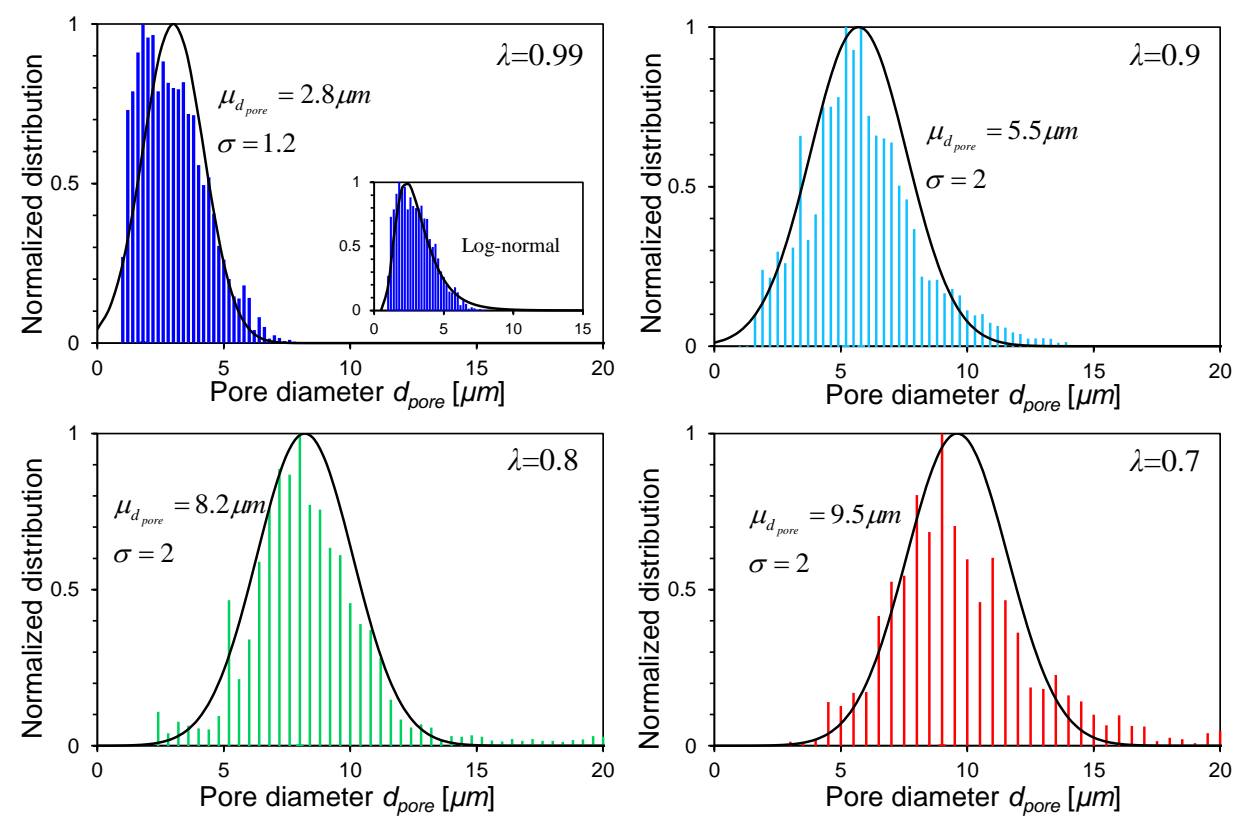

(b)
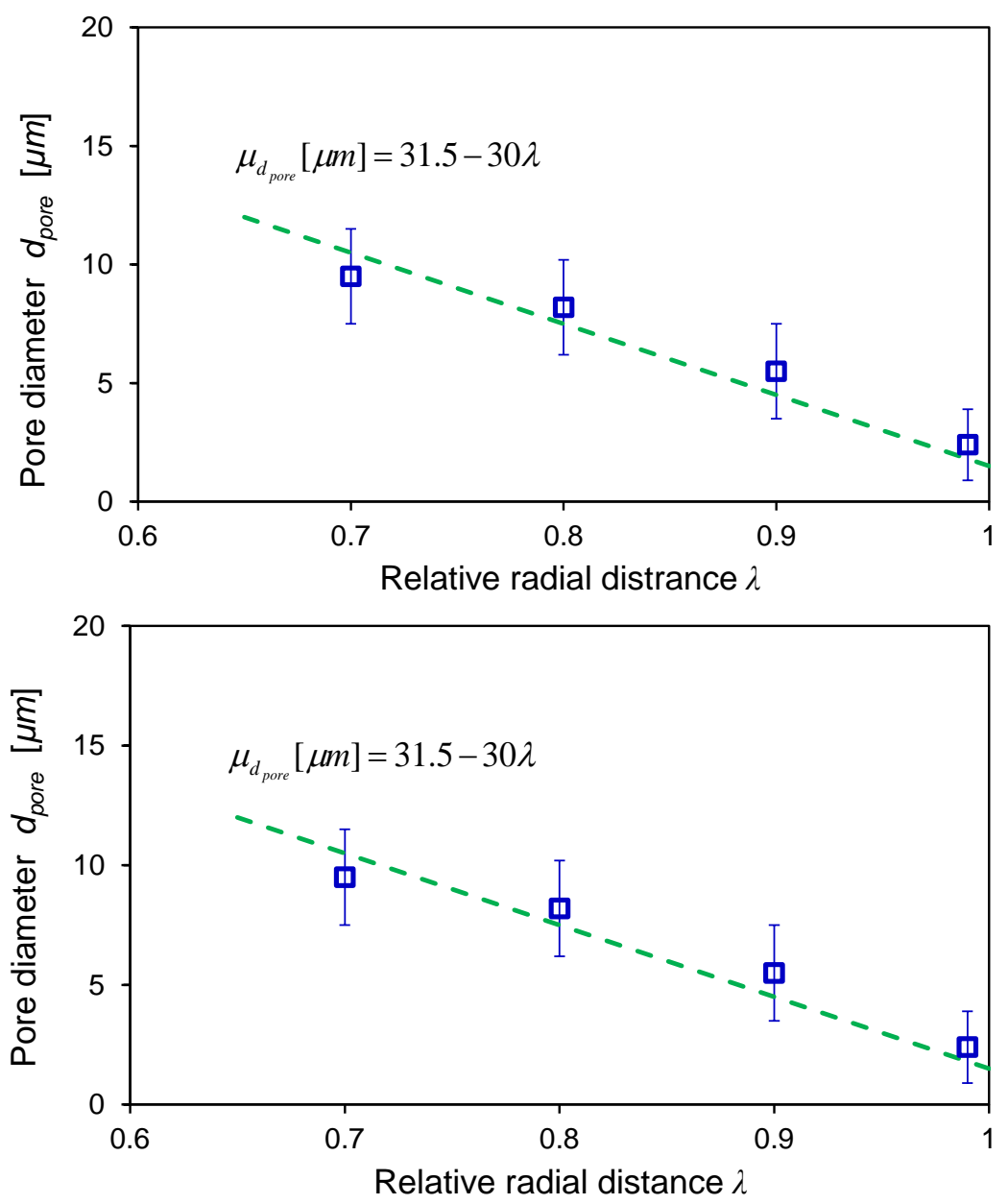

Fig. 7. Pore characteristics of the dry fiber. (a) Normally distributed pore diameter at different locations in the fiber. (b) Mean pore diameter $\mu_{\text {dpore }}[\mu \mathrm{m}]$ versus relative radial distance $\lambda$. 


\subsection{Estimation of residual IL saturation profiles}

According to the Young-Laplace equation, the capillary pressure of a pore, $\Delta u$, can be calculated as:

$\Delta u=\frac{4 \gamma \cos \theta}{d_{\text {pore }}}$

where $\theta$ is the contact angle (assumed $0^{\circ}$ herein) and $\gamma$ is the surface tension of the IL $(\gamma=0.0312 \mathrm{~N} / \mathrm{m}$ for $\left[\mathrm{C}_{6} \mathrm{mim}\right]\left[\mathrm{Tf}_{2} \mathrm{~N}\right]$ at $25^{\circ} \mathrm{C}$ ) [5]. A smaller pore size will have higher capillarity to hold the IL within the pores as gas flows through the fiber. Additionally, the pore diameter at any given radial location $\lambda$ of the hollow fiber SILMs is mostly normally distributed:

$f\left(d_{\text {pore }}\right)=\frac{1}{\sigma \sqrt{2 \pi}} e^{-\left(\frac{d_{\text {pore }}-\mu}{\sqrt{2} \sigma}\right)^{2}}$,

with $\mu[\mu \mathrm{m}]=31.5-30 \lambda$ and $\sigma=2$. Thus, the corresponding capillarity $\Delta u$ holding the IL within the pores also follows a normal distribution $f(\Delta u)$, with mean $\mu_{\Delta u}[\mathrm{MPa}]=4 \gamma /(31.5-30 \lambda)$ and $\sigma=2$.

As the cleaning gas (nitrogen in this study) flushes through the fiber bore, it creates a pressure drop that can be approximated as $\Delta P_{g}=\rho v^{2} / 2$, where $\rho$ and $v$ are the density and velocity of the gas through a tube (i.e. the Venturi effect, see also Figure 8a). Assuming the ideal gas law, the gas density is $\rho$ $=P_{i n j} \rho_{a t m}$, where $P_{i n j}$ is the injection pressure of nitrogen in bar and $\rho_{a t m}=1.25 \mathrm{~kg} / \mathrm{m}^{3}$ is the density of nitrogen at atmospheric pressure. The gas velocity is computed as $v=q / A$, where $q$ is the flux of the injected nitrogen into the fiber and $A$ is the inner cross sectional area of the fiber. Thus,

$\Delta P_{g}=\frac{P_{i n j} \rho_{a t m}}{2}\left(\frac{q}{A}\right)^{2}$.

Therefore, at any given cleaning gas pressure $P_{i n j}$ and flux $q$, the IL will be displaced in pores with capillarity $\Delta u<\Delta P_{g}$ but remains in pores with higher capillarity $\Delta u>\Delta P_{g}$ (refer to Figure 8b). Therefore, the profile of residual IL saturation $S_{r}$ of the fiber after being cleaned by gas with pressure $P_{g}$ can be estimated as

$S_{r}(\lambda)=1-\int_{0}^{P_{g}} f(\Delta u) d \xi=\frac{1}{2}\left[1-\operatorname{erf}\left(\frac{\Delta P_{g}-\mu_{\Delta u}}{\sigma \sqrt{2}}\right)\right]$.

Based on this analysis, the residual IL saturation profiles in the fiber from nitrogen flushing with different fluxes are shown in Figure 8c. Although this is an idealized case of a system with complex morphological features and gas flow that has some error between experiment and model, it roughly approximates the portion of pores that retain IL after the cleaning procedure and captures the general trend: a higher gas flux results in less remaining IL and a thinner selective layer. Too much gas flux, however, will ultimately lead to a defective selective layer. 
(a)

(c)

(b)
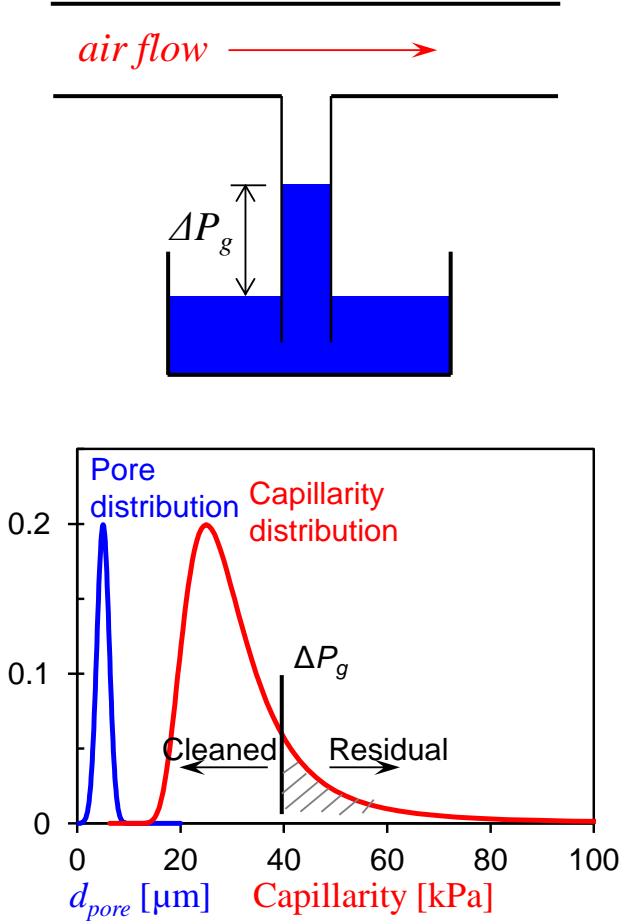

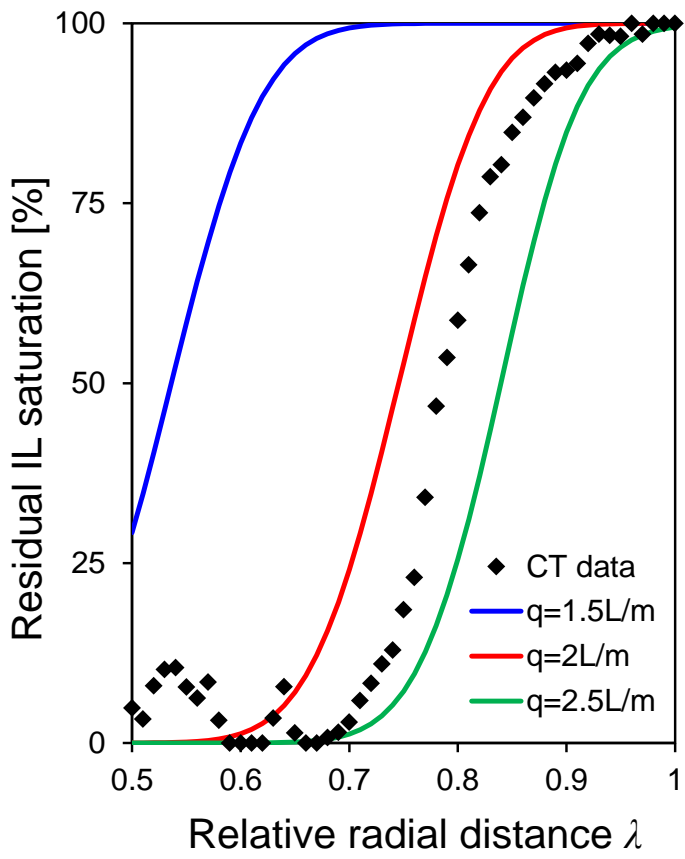

Fig. 8. Estimate of the residual IL saturation after flushing nitrogen through the hollow fiber bore. (a) Illustration of high speed gas flowing through a tube to create a pressure drop. (b) Only the IL in pores with capillarity $\Delta u$ higher than the pressure drop $\Delta P_{g}$ will remain after the cleaning procedure. (c) Estimated saturation profiles using various gas flow rates.

\section{Conclusion}

The microfocus X-ray CT has been used to investigate a hollow fiber SILM at different conditions: dried, saturated by IL, and after being cleaned by flowing of gas through the fiber bore. The CT scanning gives a 3D spatial structure of different phases of the hollow fiber (air, ionic liquid, and polymer) with micron scale resolution. By this method it is possible to resolve the distribution of IL within the fiber cross section non-destructively. Further processing of the CT images can provide critical information such as pore characteristics, pore distribution, and residual IL distribution in the hollow fibers. Through this analysis, we have found that:

- The average pore diameter in this hollow fiber decreases almost linearly with increasing relative radial distance $\lambda$. The size of macro pores is normally distributed with identical standard deviation of $\sigma=2 \mu \mathrm{m}$; while the pore size distribution of meso-pores follows a log-normal trend with less scattering.

- The porosity in the macro-porous region is $v \approx 0.45$ and that in the meso-porous region is $v \approx$ 0.3 ; thus, the macro-porous region near the fiber bore has a higher porosity than meso-porous region near the fiber shell.

- It is clearly shown that flowing nitrogen gas through the fiber bore under pressure entrains and removes IL from the macro-pores but not from the meso-pores. This is analyzed by unrolling radial sections of the fiber and using threshold analysis to determine the ratio of air, IL, and polymer phases at different radial regions throughout the fiber cross-section.

- The residual IL saturation in the fiber after being purged by gas is inherently controlled by the pore size and the gas flux. Specifically, the amount of residual IL is inversely proportional to the 
pore size, the gas flux, and the gas pressure.

This technique can be used to evaluate the compatibility of various types of hollow fibers and ILs for gas separation membrane applications.

\section{Acknowledgement}

We gratefully acknowledge funding and support from the U.S. Department of Energy National Energy Technology Laboratory (NETL) under the FY14 Carbon Capture field work proposal. Sheng Dai is supported under an Oak Ridge Institute for Science and Education (ORISE) fellowship granted by NETL. This report was prepared as an account of work sponsored by an agency of the United States Government. Neither the United States Government nor any agency thereof, nor any of their employees, makes any warranty, express or implied, or assumes any legal liability or responsibility for the accuracy, completeness, or usefulness of any information, apparatus, product, or process disclosed, or represents that its use would not infringe on privately owned rights. Reference herein to any specific commercial product, process, or service by trade name, trademark, manufacturer, or otherwise does not necessarily constitute or imply its endorsement, recommendation, or favoring by the United States Government or any agency thereof. The views and opinions of authors expressed herein do not necessarily state or reflect those of the United States Government or any agency thereof. 


\section{References}

1. R.D. Noble, D.L. Gin, Perspective on ionic liquids and ionic liquid membranes, J. Membr. Sci. 369 (2011) 1 .

2. S. Wickramanayake, D. Hopkinson, C. Myers, L. Sui, D. Luebke, Investigation of transport and mechanical properties of hollow fiber membranes containing ionic liquids for pre-combustion carbon dioxide capture, J. Membr. Sci. 439 (2013) 58-67.

3. W. Lan, S. Li, J. Xu, G. Luo, Preparation and Carbon Dioxide Separation Performance of a Hollow Fiber Supported Ionic Liquid Membrane, Ind. Eng. Chem. Res. 52 (2013) 6770-6777.

4. S. Wickramanayake, D. Hopkinson, C. Myers, L. Hong, J. Feng, Y. Seol, D. Plasynski, M. Zeh, D. Luebke, Mechanically robust hollow fiber supported ionic liquid membranes for $\mathrm{CO}_{2}$ separation applications, J. Membr. Sci. 470 (2014) 52-59.

5. D. Hopkinson, M. Zeh, D. Luebke, The bubble point of flat sheet supported ionic liquid membranes, J. Membr. Sci. 468 (2014) 155-162.

6. J. Viguie, T. Savart, P. Duru, J.C. Rouch, J.C. Remigy, Characterization of 3D macrostructure of hollow fibre membranes using X-ray tomography - Effects of some spinning process conditions, J. Membr. Sci. 435 (2013) 11-20.

7. S.S. Manickam, J.R. McCutcheon, Characterization of polymeric nonwovens using porosimetry, porometry, and X-ray computed tomography, J. Membr. Sci. 407-408 (2012) 108-115.

8. J. C. Jansen, M.Macchione, E. Drioli, High flux asymmetric gas separation membranes of modified poly(ether ether ketone) prepared by the dry phase inversion technique, J. Membr. Sci. 255 (2005) 167.

9. W.J. Koros, I. Pinnau, Polymeric Gas Separation Membranes, CRC Press, Boca Raton, FL,1995.

10. S.C. Pesek, W.J. Koros, Aqueous quenched asymmetric polysulfone membranes prepared by dry/wet phase separation, J. Membr. Sci. 81 (1993) 71-88.

11. N.A. Dyson, X rays in atomic and nuclear physics, Cambridge University Press, Cambridge, UK, 1973.

12. E.C. McCullough, Photon attenuation in computed tomography, Medical Physics, 2(6) (1975), 307320.

13. G. Herman, Image reconstruction from projections: the fundamentals of computerized tomography, Academic, New York, NY, 1980.

14. W. Rasband, ImageJ, U.S. National Institutes of Health, Bethesda, Maryland, USA, 1997.

15. A.C. Kak, M. Slaney, Principles of computerized tomographic imaging, Society for Industrial and Applied Mathematics, Philadelphia, Pennsylvania, USA, 2001.

16. M.D. Abràmoff, P. J. Magalhães, S. J. Ram (2004), Image processing with ImageJ, Biophotonics International, 11(7) (2004) 36-43.

17. I. Bankman, Handbook of medical image processing and analysis, Academic Press, Burlington, MA, USA, 2008.

18. R.H. Bates, M.J. McDonnell, Image restoration and reconstruction, Clarendon Press, Oxford, UK, 1986.

19. J.C. Russ, R.P. Woods, The image processing handbook, Journal of Computer Assisted Tomography, 19(6) (1995), 979-981.

20. ASTM-E1935-97, Standard test method for calibrating and measuring CT density, ASTM, 2013.

21. N. Otsu, A threshold selection method from gray-level histograms, Automatica, 11(285-296), (1975) 23-27.

22. P.K. Sahoo, S. Soltani, A. K. Wong, A survey of thresholding techniques, Computer vision, graphics, and image processing, 41(2) (1988), 233-260. 